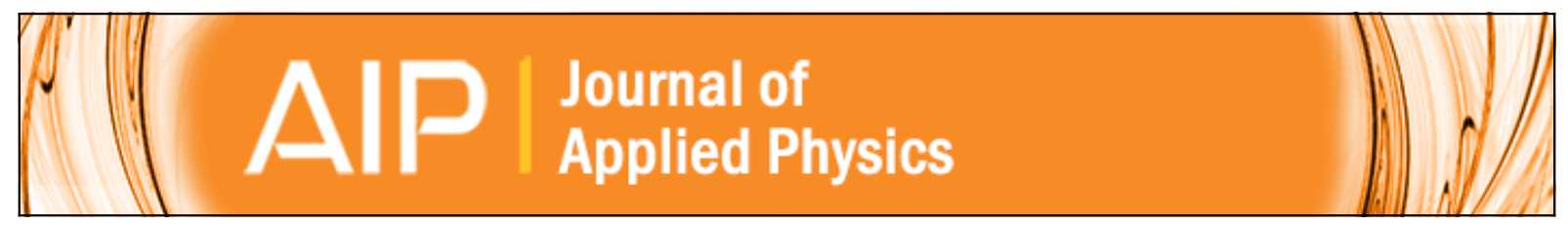

\title{
Dynamics of self-driven microcantilevers
}

A. Passian, G. Muralidharan, S. Kouchekian, A. Mehta, S. Cherian, T. L. Ferrell, and T. Thundat

Citation: Journal of Applied Physics 91, 4693 (2002); doi: 10.1063/1.1452771

View online: http://dx.doi.org/10.1063/1.1452771

View Table of Contents: http://scitation.aip.org/content/aip/journal/jap/91/7?ver=pdfcov

Published by the AIP Publishing

\section{Articles you may be interested in}

Microcantilever dynamics in tapping mode atomic force microscopy via higher eigenmodes analysis J. Appl. Phys. 113, 224302 (2013); 10.1063/1.4808446

Forced oscillations with continuum models of atomic force microscopy

AIP Conf. Proc. 1493, 230 (2012); 10.1063/1.4765494

Self-driven soft imaging in liquid by means of photothermal excitation

J. Appl. Phys. 110, 114315 (2011); 10.1063/1.3665396

Dynamical systems analysis for polarization in ferroelectrics

J. Appl. Phys. 100, 114106 (2006); 10.1063/1.2388124

Control of microcantilevers in dynamic force microscopy using time delayed feedback

Rev. Sci. Instrum. 77, 053703 (2006); 10.1063/1.2200747

\section{MIT LINCOLN} LABORATORY CAREERS

Discover the satisfaction of innovation and service to the nation
- Space Control

- Air \& Missile Defense

- Communications Systems \& Cyber Security

- Intelligence, Surveillance and

Reconnaissance Systems

- Advanced
Electronics
- Tactical Systems
- Homeland
Protection
- Air Traffic Control

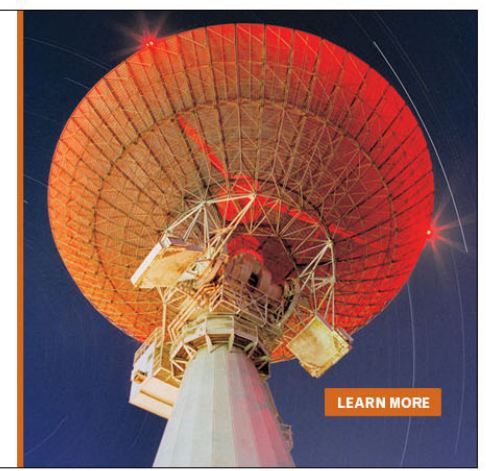




\title{
Dynamics of self-driven microcantilevers
}

\author{
A. Passian ${ }^{\text {a) }}$ and G. Muralidharan \\ Oak Ridge National Laboratory, Bethel Valley Road, Oak Ridge, Tennessee 37830
}

S. Kouchekian

Department of Mathematics, Virginia Polytechnic Institute and State University, Blacksburg, Virginia 240610123

A. Mehta, S. Cherian, T. L. Ferrell, and T. Thundat

Oak Ridge National Laboratory, Bethel Valley Road, Oak Ridge, Tennessee 37830

(Received 22 October 2001; accepted for publication 27 December 2001)

\begin{abstract}
The small amplitude thermal vibrations of the microcantilever of an atomic force microscope can be enhanced via a delayed feedback system. This is verified experimentally for a triangular cantilever, and modeled theoretically as a boundary value problem resulting in a second order functional differential equation for the temporal behavior of the cantilever. The eigenvalues of the resulting delay differential equation describing the transverse vibrations of the cantilever are calculated and analyzed. These values are compared with the corresponding resonant frequencies predicted by a point mass model and with the experimentally observed values. (C) 2002 American Institute of Physics. [DOI: 10.1063/1.1452771]
\end{abstract}

\section{INTRODUCTION}

The atomic force microscope (AFM), since its invention by Binnig et al., ${ }^{1}$ has been extensively used in imaging, chemical and biological sensing, and providing surface characteristics in various fields of applications. ${ }^{2-4}$ The probing capability of this device is based on the interaction of an oscillating microcantilever within the nanometer, angstrom, or contact ranges with the surface attributes of the sample under study, which generates a measurable signal that reveals electric and magnetic properties of the sample surfaces. ${ }^{5}$ The dynamics of the cantilever motion are of great interest and have been studied by many authors. Chen et al. ${ }^{5}$ calculated the fundamental and harmonic resonance frequencies for $\mathrm{V}$ shaped and rectangular cantilevers using a variational method to solve the beam equation. Sader ${ }^{6}$ discusses the hydrodynamic aspects and the resonant frequencies of a cantilever beam with uniform arbitrary cross section that is immersed in a viscous fluid and is driven by an arbitrary force. The vibrational dynamics of free and surface coupled cantilevers have been studied using equation of motion for flexural vibrations and the simplified first mode approximation (point-mass model) and compared with experimental observations for cantilevers. ${ }^{7-9}$

With the advent of noncontact force and tapping mode microscopy the dynamics of a cantilever driven in a feedback loop has attracted a lot of attention. Albrecht et al. ${ }^{10}$ used a frequency modulation technique, where the cantilever served as the frequency determining element of the oscillator, to improve the sensitivity without restricting the bandwidth or the dynamic range. Dürig et al. ${ }^{11}$ using the cantilever as a resonator in an active feedback circuit for sensing interaction forces in dynamic force microscopy showed the tracking os-

\footnotetext{
a) Author to whom correspondence should be addressed; electronic mail: passian@utk.edu
}

cillator technique to be superior to the direct feedback method, allowing one to differentiate between conservative and dissipative interactions.

Recently, Mehta et al. ${ }^{12}$ studied the "Brownian" motion of the microcantilevers induced by ambient thermal fluctuations and utilized that in a feedback system to improve the $Q$ factor of the cantilever in air and water by several orders of magnitude. Similarly, Muralidharan et al. ${ }^{13}$ showed that small amplitude thermal vibrations of a microcantilever of triangular shape can be enhanced by engaging a delayed feedback mechanism. In their work, the microcantilever was modeled as a system with a single degree of freedom (DOF) consisting of a mass $m$, a spring (spring constant $k$ ), and a dashpot (viscous damping constant $\gamma$ ), heretofore, referred to as the "point mass model." The effect of the feedback was incorporated by convoluting the current location of the point mass with that of an earlier time. The feedback was characterized using a time delay $\tau$ and a gain $G$. The point mass model has been further investigated, and experimental work has been carried out ${ }^{14}$ where the dynamics of a geometrically different cantilever was studied. Results of the investigations show that although the point mass model was successful in rationalizing conditions for which amplification succeeded, it lacked the desired predictive capabilities. Furthermore, it was noticed that the predicted resonant frequencies for the combinations of parameters $(\tau$ and $G)$ used in the experiments were different from the experimentally observed values for the resonant frequencies. It is believed that this discrepancy is due to treating the system as a discrete one with a single DOF rather than a continuous one.

The primary purpose of this work is to remove the above-mentioned deficiency and analyze the vibrational behavior of the cantilever using the beam equation which takes into account the finite spatial extent of the microcantilever. We discuss delay, gain, and amplification intervals in which resonance is plausible, and address further work related to 


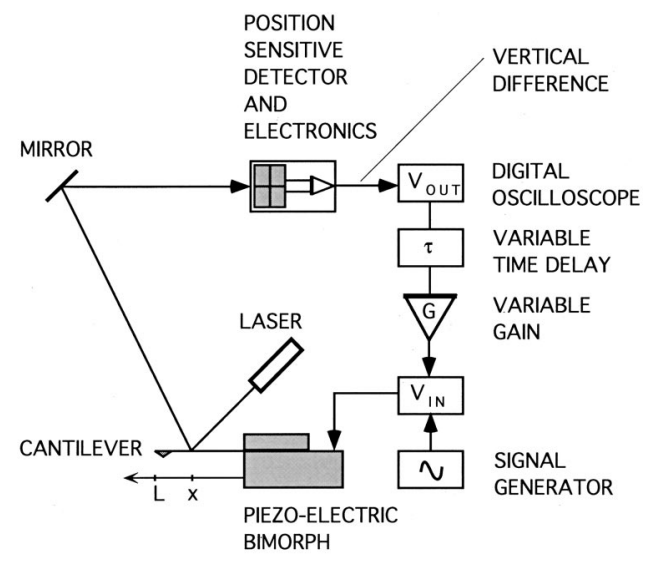

FIG. 1. Schematics of the experimental setup. The variable time delay and gain modules are used to control the feedback process.

such feedback mechanisms. Section II outlines the experimental procedure to accomplish a delayed feedback. In Sec. III, the mathematical model based on the beam equation for the cantilever is explained, and relevant equations are derived. Section IV presents the results of the numerical work which is discussed and summarized in Sec. V where comparisons are made with the point mass model and the experimental results. A conclusion is given in Sec. VI.

\section{EXPERIMENTAL METHOD}

The experimental configuration is depicted in Fig. 1. We closely follow the procedure outlined in Ref. 13. The cantilevers used for the study were commercially available contact levers that are triangular in shape and made of silicon nitride. The nominal length and width of the cantilevers are 100 and $20 \mu \mathrm{m}$, respectively. These are mounted in a commercially available cantilever holder used for tapping mode AFM with an in-built piezoelectric bimorph that drives the cantilever. The whole assembly is placed inside an AFM head containing the laser source and a two or a four quadrant-position sensitive detector. The vertical difference output from the detector (and associated electronics) is amplified and constitutes $V_{\text {out }}$. This output signal is then routed through a variable time delay circuit that causes a delay $\tau$ and a variable gain amplifier with an amplification factor $G$ before it is applied to the piezoelectric bimorph. For future reference, we call this input voltage $V_{\text {in }}$. Both the voltages $V_{\text {out }}$ and $V_{\text {in }}$ are monitored with a high-speed digital oscilloscope capable of sampling at the rate of $100 \mathrm{MHz}$ (minimum of $10 \mathrm{~ns}$ sampling interval) interfaced to a computer. For studying the response of the cantilever to a specific shape of input signal, the experimental setup allowed the output from a spectrum generator to be applied directly to the piezoelectric bimorph as shown in Fig. 1.

\section{DYNAMICAL MODEL}

The transverse vibrations of a cantilever exposed to an arbitrary resistive force $R(x, t)$ and an arbitrary driving force $F(x, t)$ can be described by the partial differential equation ${ }^{15}$

$$
E I \frac{\partial^{4} W(x, t)}{\partial x^{4}}+\mu \frac{\partial^{2} W(x, t)}{\partial t^{2}}+R(x, t)=F(x, t),
$$

where the positive constants $E, I$, and $\mu$ are Young's modulus, area moment of inertia, and the mass per unit length at $x$, respectively. It should be understood that $R(x, t)$, and $F(x, t)$ are forces per unit length of the cantilever. For an undriven cantilever, excited by an impulse at $t=0$, the amplitude of the subsequent oscillations under atmospheric pressure and room temperature, will decay as a function of time due to damping. Sader ${ }^{6}$ considers the hydrodynamic damping due to motion in an incompressible viscous fluid, and suggests further that the same arguments hold for gases provided certain conditions are satisfied. Here, due to the low density of air compared to that of a continuum, and the fact that air is compressible, we can write the damping due to a viscoelastic resistive force as ${ }^{15}$

$$
R(x, t)=k W(x, t)+\gamma \frac{\partial W(x, t)}{\partial t},
$$

where $k$ and $\gamma$ are positive constants. The visco-elastic force as represented by Eq. (2) has the form of the Kelvin-Voigt element, i.e., a parallel (as opposed to a series) combination of the basic elastic and viscous elements. ${ }^{16}$ Thus, $R(x, t)$ will here designate the damping of oscillations both due to the viscosity of the surrounding air and to internal losses in the cantilever. Furthermore, referring to Ref. 13, we assume the following delayed feedback form:

$$
F(x, t)=-k^{\prime} G W(x, t-\tau),
$$

where the dimensionless constant $|G|$ is the gain, the constant $\tau \geqslant 0$ represents the total time delay, and $k^{\prime}$ is the effective spring constant which will be used as a proportionality constant. $G$ can be related to the electronic gain as well as the relationship between the voltage applied to the piezoelectric bimorph and the magnitude of the resulting displacement of the cantilever as outlined in Ref. 13. In contrast to the previous work where $G$ was assumed to be positive, we now recognize that $G$ can either be positive or negative and varies with the cantilever holder. This is due to the fact that for a positive voltage $V_{\text {in }}$ applied to the bimorph, the piezoelectric bimorph can either move in the same direction as the original motion of the cantilever or in the opposite direction depending on the orientation of the bimorph in the microcantilever holder. Mathematically, this corresponds to an uncertainty in the sign of $G$ due to an uncertainty in the sign of $\chi$ in the following equation:

$$
z_{p}(t)=\chi V_{\text {in }}(t),
$$

where $\chi$ is a constant related to the response of the bimorph. ${ }^{13}$ In formulating the differential equation that describes the motion of the microcantilever in the presence of feedback, we make an important assumption in that although the measured signal from the reflected laser beam arises only due to the vertical deflection of the cantilever at the illuminated spot with a finite diameter $\delta<L$ along the cantilever, as shown schematically in Fig. 1, we take the delayed feedback signal as that representing the vibrational state of the entire cantilever at a previous time. In particular, on the pre- 
interval $[-\tau, 0]$, we propose the use of a linear combination of the dominating undriven eigenmodes $\phi(x, t)$ of the cantilever as the prefunction. In this section we also present the explicit form of $\phi(x, t)$. Thus, making the change of variable $x \rightarrow x / L$, the motion of a cantilever of length $L$, driven by the feedback Eq. (3), can be described by the following delay partial differential equation:

$$
\begin{aligned}
\frac{E I}{L^{4}} & \frac{\partial^{4} W(x, t)}{\partial x^{4}}+\mu \frac{\partial^{2} W(x, t)}{\partial t^{2}}+k W(x, t)+\gamma \frac{\partial W(x, t)}{\partial t} \\
& =-k^{\prime} G W(x, t-\tau),
\end{aligned}
$$

subject to the standard fixed-free beam boundary conditions

$$
W(0, t)=W_{x}(0, t)=W_{x x}(1, t)=W_{x x x}(1, t)=0,
$$

and the initial condition

$$
W(x, t)=\phi(x, t) \text { on }[-\tau, 0],
$$

for each $x \in[0, L]$. We note that when $\tau=0$, the condition in Eq. (7) reduces to the standard initial condition $W(x, 0)$ $=f(x)$, whereas for finite delays $\tau \ll 1$, the motion of the lever depends explicitly on it's dynamic history. Then with $\tau=0$, and the particular choice of the initial condition $f(x)$ $=\phi(x, 0)$, the problem can be solved exactly, since the undriven eigenfunctions are smooth and form an orthogonal set. This exact solution can then be compared with the solutions to the delay equation (5) in the limit $\tau \rightarrow 0$, as a validation check.

Due to the particular form of the right hand side of Eq. (5), and without resorting to Fourier space, we separate the differential equation in Eq. (5) via the ansatz $W(x, t)$ $=X(x) T(t)$, and require that the separation constant $\mathcal{K}>0$ (see Appendix) and obtain for the spatial dependence, the equations

$$
\left\{\begin{array}{l}
X^{(4)}(x)-\lambda^{4} X(x)=0 \\
X(0)=X^{\prime}(0)=X^{\prime \prime}(1)=X^{\prime \prime \prime}(1)=0,
\end{array}\right.
$$

where

$$
\lambda^{4}=\frac{L^{4} \mathcal{K}}{E I},
$$

and

$$
\mu T^{\prime \prime}(t)+\gamma T^{\prime}(t)+(\mathcal{K}+k) T(t)=-k^{\prime} G T(t-\tau)
$$

for the temporal behavior. Equation (8) is standard. The set of eigenfunctions $\left\{X_{n}(x)\right\}_{1}^{\infty}$ satisfying Eq. (8) form an orthonormal set, and are explicitly given by

$$
\begin{aligned}
X_{n}(x)= & \cosh \lambda_{n} x-\cos \lambda_{n} x \\
& -C\left(\lambda_{n}\right)\left(\sinh \lambda_{n} x-\sin \lambda_{n} x\right),
\end{aligned}
$$

where

$$
C\left(\lambda_{n}\right)=\frac{\cosh \lambda_{n}+\cos \lambda_{n}}{\sinh \lambda_{n}+\sin \lambda_{n}},
$$

and

$$
\mathcal{K}_{n}=\frac{\lambda_{n}^{4} E I}{L^{4}},
$$

and $0<\lambda_{1}<\lambda_{2}<\lambda_{3}<\ldots$ are the positive roots of the implicit equation

$$
1+\cos \lambda \cosh \lambda=0 .
$$

We now consider the undriven case, i.e., $F(x, t) \equiv 0$ in Eq. (1). Equation (9) then describes a linear homogeneous differential equation for which the general solution takes on the form

$T_{n}(t)=\left(a_{n} \cos \omega_{n} t+b_{n} \sin \omega_{n} t\right) e^{-(\gamma / 2 \mu) t}, \quad n=1,2, \cdots$,

where $a_{n}$ and $b_{n}$ are arbitrary complex constants, and $\omega_{n}$ denotes the $n$th radial frequency of the beam and is given by

$$
\omega_{n}=\sqrt{\frac{1}{\mu}\left(\mathcal{K}_{n}+k\right)-\left(\frac{\gamma}{2 \mu}\right)^{2}},
$$

which is real for all $n$ assuming the damping is not too large. Finally, as discussed earlier, in view of Eqs. (10), (11), (12), (14), and (15), we are able to express the prefunction $\phi(x, t)$ as

$$
\phi(x, t)=\sum_{n=1}^{N} A_{n} X_{n}(x) e^{-(\gamma / 2 \mu) t} \cos \left(\omega_{n} t+\theta_{n}\right),
$$

where $A_{n} \cos \left(\omega_{n} t+\theta_{n}\right)$ is the $n$th harmonic of amplitude $\left|A_{n}\right|$ and initial phase $\theta_{n}$. In most practical cases, it would suffice to take the first few terms in the sum, i.e., $N=2$ or $N=3$.

Next we turn our attention back to Eq. (9). Corresponding to each fixed mode $n=1,2 \cdots$, this equation yields a delay differential equation of the form

$\mu T_{n}^{\prime \prime}(t)+\gamma T_{n}^{\prime}(t)+\left(\mathcal{K}_{n}+k\right) T_{n}(t)=-k^{\prime} G T_{n}(t-\tau)$.

One natural way to solve Eq. (17) is to seek a solution of the form $g(t)=e^{\beta_{n} t}$, where $\beta_{n}$ is a complex number. It turns out, by direct substitution, that $g(t)$ solves Eq. (17) if and only if $\beta_{n}$ satisfies the following equation:

$$
\beta_{n}^{2}+\frac{\gamma}{\mu} \beta_{n}+\frac{1}{\mu}\left(\mathcal{K}_{n}+k\right)=-\frac{k^{\prime}}{\mu} G e^{-\beta_{n} \tau} .
$$

If $\left\{\beta_{n, m}\right\}$ denotes either the finite or infinite sequence of distinct roots of Eq. (18), then the general solution of Eq. (17) is given by

$$
T_{n}(t)=\sum_{m} p_{n, m}(t) e^{\beta_{n, m} t},
$$

where $p_{n, m}(t)$ is an arbitrary polynomial of degree less than the multiplicity of the root $\beta_{n, m}$. In the case of infinitely many roots, we have to also impose conditions which ensure the convergence of the infinite sum (see Ref. 17). It is also of interest to note that in most cases the transcendental equation (18) has infinitely many roots. Finally, the general solution to the beam's equation (5) with the boundary conditions Eq. (6) can be written as

$$
W(x, t)=\sum_{n} \sum_{m} X_{n}(x) p_{n, m}(t) e^{\beta_{n, m} t},
$$


where the coefficients of each polynomial $p_{n, m}(t)$ should be determined by the initial condition. Although it is only for very special cases in which one can explicitly determine $W(x, t)$, Eq. (20) provides us with a good understanding of the nature of the solutions for the beam's equation subject to a delayed driving force. There is also another method of piecewise continuation of the solution, which avoids the series solution discussed above. According to this approach, one extends the solution of Eq. (5), with increasing $t$, from each interval of length $\tau$ to another interval of the same length starting at $[-\tau, 0]$. A more detailed theoretical analysis with respect to the exact solution of the partial differential equation (5) subject to the boundary and initial conditions Eqs. (6) and (7) is beyond the main purpose of this article. These results will be presented in a forthcoming article. In this article, however, we are mainly concerned with those values of the delay time $\tau$ and the gain $G$, which result in a successful amplification of the signal with frequencies close to the normal frequencies $\omega_{n}$. More precisely, we seek a solution $\beta_{n}$ to Eq. (18) such that the real part of $\beta_{n}$ is positive while its imaginary part is close to $\omega_{n}$. First of all observe that since $\mu, \gamma$ are both positive constants, Eq. (18) will have real/complex roots for $G<0$ (depending on the magnitude of $G$ ), or no real roots for $G>0$. This explains our complex substitution for $\beta_{n}$. Furthermore, since all the coefficients of Eq. (18) are real, the roots will appear as pairs of conjugate numbers. Substituting $\beta_{n}=\alpha_{n}+i\left(\omega_{n}+\epsilon_{n}\right),\left|\epsilon_{n}\right|$ $<\omega_{n}$ in Eq. (18) and using Eq. (15), we find the following equations for $\alpha_{n}$ :

$\left\{\begin{array}{l}{\left[\left(\alpha_{n}+\tilde{\gamma}\right)^{2}-\epsilon_{n}\left(\epsilon_{n}+2 \omega_{n}\right)\right] e^{\alpha_{n} \tau}=-\tilde{k} G \cos \left[\left(\omega_{n}+\epsilon_{n}\right) \tau\right]} \\ 2\left(\alpha_{n}+\tilde{\gamma}\right)\left(\epsilon_{n}+\omega_{n}\right) e^{\alpha_{n} \tau}=\tilde{k} G \sin \left[\left(\omega_{n}+\epsilon_{n}\right) \tau\right],\end{array}\right.$

where

$$
\tilde{\gamma}=\frac{\gamma}{2 \mu}
$$

and

$$
\tilde{k}=\frac{k^{\prime}}{\mu} .
$$

From Eq. (21) and the requirements $\alpha_{n}>0,\left|\epsilon_{n}\right|<\omega_{n}$, it follows at once that the delay time $\tau$ is bounded within the bands $\left(2 p+\frac{1}{2}\right) \pi<\left(\omega_{n}+\epsilon_{n}\right) \tau<(2 p+1) \pi$, for $G>0$, and the higher bands $\left(2 p+\frac{3}{2}\right) \pi<\left(\omega_{n}+\epsilon_{n}\right) \tau<2(p+1) \pi$, for $G<0$, where $p=0,1,2, \cdots$ is an integer that specifies the location of the allowed band. Finally, after some algebra, and taking into account the restrictions just mentioned, one can conclude that Eq. (21) has the unique solution

$$
\begin{aligned}
\alpha_{n}\left(\epsilon_{n}\right)= & -\tilde{\gamma}-\left(\epsilon_{n}+\omega_{n}\right) \cot \left[\left(\omega_{n}+\epsilon_{n}\right) \tau\right] \\
& +\left\{\left(\epsilon_{n}+\omega_{n}\right)^{2} \cot ^{2}\left[\left(\omega_{n}+\epsilon_{n}\right) \tau\right]\right. \\
& \left.+\epsilon_{n}\left(\epsilon_{n}+2 \omega_{n}\right)\right\}^{1 / 2},
\end{aligned}
$$

if and only if the delay $\tau$ and gain $G$ can be chosen in such a way that they satisfy the conditions
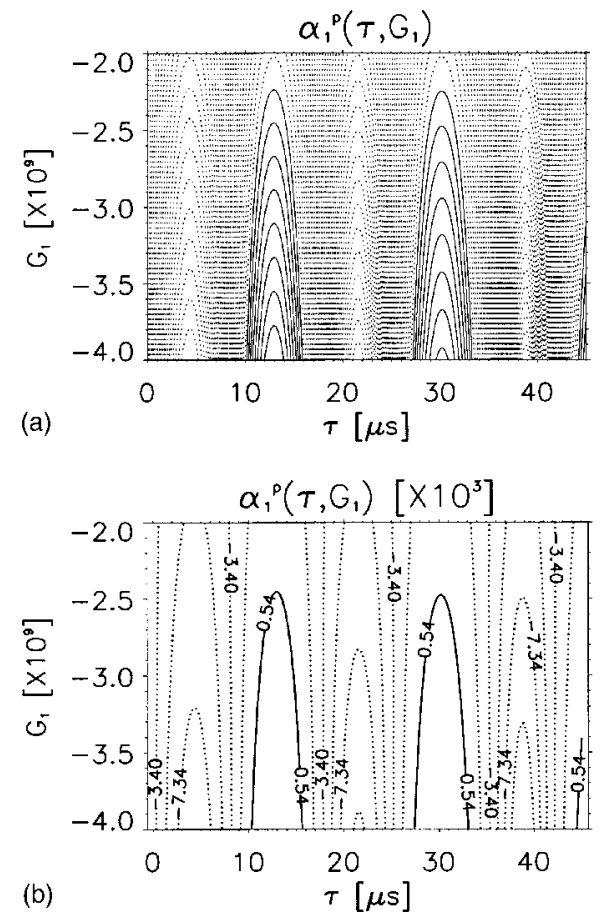

FIG. 2. (a) Variation in $\alpha_{1}^{p}\left(\tau, G_{1}\right)$ as calculated from Eq. (21) for negative values of $G$. The allowed delays are restricted to the values in the region shown by the solid lines. The dotted lines correspond to negative values of $\alpha_{1}^{p}\left(\tau, G_{1}\right)$ for which the oscillations will be damped out rather than amplified. (b) Magnitude of $\alpha_{1}^{p}\left(\tau, G_{1}\right)$ for some selected contours.

$\begin{cases}\left(2 p+\frac{1}{2}\right) \pi<\left(\omega_{n}+\epsilon_{n}\right) \tau<(2 p+1) \pi, & \text { for } G>0 \\ \left(2 p+\frac{3}{2}\right) \pi<\left(\omega_{n}+\epsilon_{n}\right) \tau<2(p+1) \pi, & \text { for } G<0,\end{cases}$

for some integer $p \geqslant 0$, and

$$
G_{n}=G\left(\epsilon_{n}\right)=\frac{2\left(\alpha_{n}+\tilde{\gamma}\right)\left(\epsilon_{n}+\omega_{n}\right)}{\tilde{k} \sin \left[\left(\omega_{n}+\epsilon_{n}\right) \tau\right]} e^{\alpha_{n} \tau}
$$

bearing in mind that the $\alpha_{n}$ used in Eq. (25) is given by Eq. (23). Alternatively, in Eq. (21) we can treat the delay $\tau$ and the gain $G$ as parameters and solve for $\alpha$ and $\epsilon_{n}$ as shown in Sec. IV.

\section{RESULTS}

Figures 2 and 3 show the values of $\alpha_{n}$ as a function of time delays $\tau$ and $G_{n}$ obtained from the solutions of the system of equations (21). The factor $\gamma / 2 m=2.8 \times 10^{3} \mathrm{~s}^{-1}$ related to the damping of the motion of the microcantilever was obtained by analyzing the decay of the amplitude of the oscillations obtained by subjecting the microcantilever to a square wave form as outlined in Ref. 13. As a consequence of requiring an amplification of the cantilever's oscillations $\left(\alpha_{n} \geqslant 0\right)$, the range of useful delay times are restricted to certain bands as shown in Eq. (24) and as shown by the solid lines in Figs. 2(a) and 2(b) for negative values of $G$ and Figs. 3(a) and 3(b) for positive values of $G$. Shown in these figures are the first few permitted delay bands corresponding to $p$ $=0,1,2$. The delay times within the allowed bands are inter- 

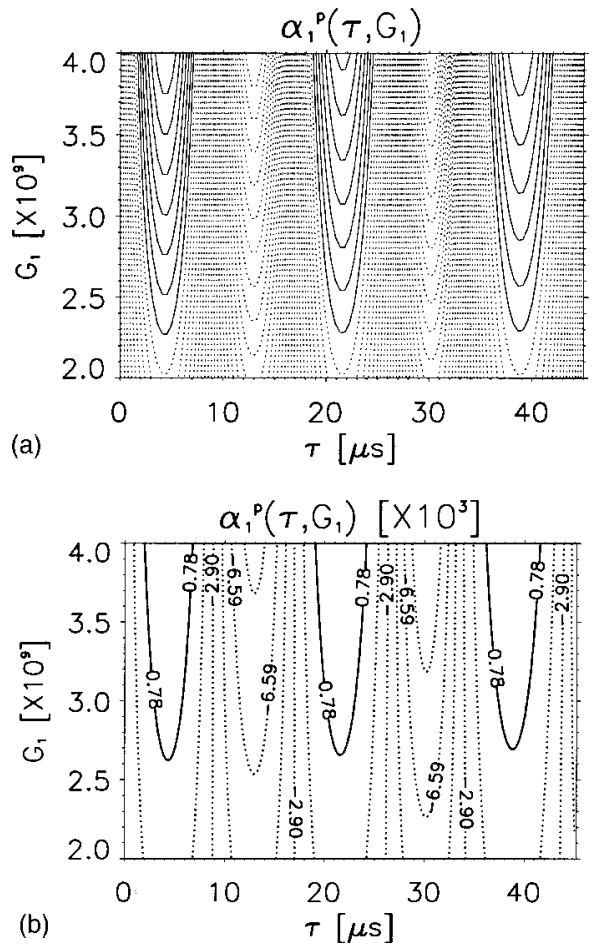

FIG. 3. (a) Variation in $\alpha_{1}^{p}\left(\tau, G_{1}\right)$ as calculated from Eq. (21) for positive values of $G$. The allowed delays are restricted to the values in the region shown by the solid lines. The dotted lines correspond to negative values of $\alpha_{1}^{p}\left(\tau, G_{1}\right)$ for which the oscillations will be damped out rather than amplified. (b) Magnitude of $\alpha_{1}^{p}\left(\tau, G_{1}\right)$ for some selected contours.

preted so that the actual physical delays $\tau_{p}$ are obtained for $\tau>T_{n}$ via $\tau_{p}=\tau-p T_{n}$ for $p=0,1,2, \cdots$, where $T_{n}=2 \pi \omega_{n}^{-1}$ is the $n$th observed period of the undriven cantilever oscillation. Specifically, for the fundamental resonant frequency considered here, $n=1$ and $\tau_{p}=\tau-17.24 \times p(\mu \mathrm{s})$ where $p$ $=0,1,2, \cdots$. For the sake of simplicity, the value of the gain $G_{1}$ has been obtained by setting

$$
\tilde{k}=1,
$$

in Eq. (22).

An important result desired from the calculations was the frequency of vibration of the cantilevers. Since the experimentally observed fundamental frequencies of the feedback-driven cantilever were not significantly different from the fundamental resonant frequency of the undriven cantilever $\omega_{1}=2 \pi f_{1}$, the magnitude of $\epsilon_{1}=\epsilon$ in Eqs. (21) was chosen so that it would allow for solutions in the vicinity of $\omega_{1}$. The allowed values of $(2 \pi)^{-1} \epsilon$ calculated using the beam equation are shown as functions of $\tau$, and $G_{1}$ in Figs. 4(a) and 4(b) for negative and positive values of $G_{1}$, respectively. The experimental values of $\tau$ and $\left|G_{1}\right|$ for which amplification was observed are labeled by numbers $0-10$ in Fig. 4 , and shown in Table I. Also shown in Table I are the measured values for frequency $f$ and $(2 \pi)^{-1} \epsilon$. It should be understood here that the calculated frequencies are given by $\omega=\omega_{1}+\epsilon(\mathrm{kHz})$ and hence, $f=f_{1}+(2 \pi)^{-1} \epsilon$. The range of $\epsilon$ can be varied in order to examine other frequency regions of interest.
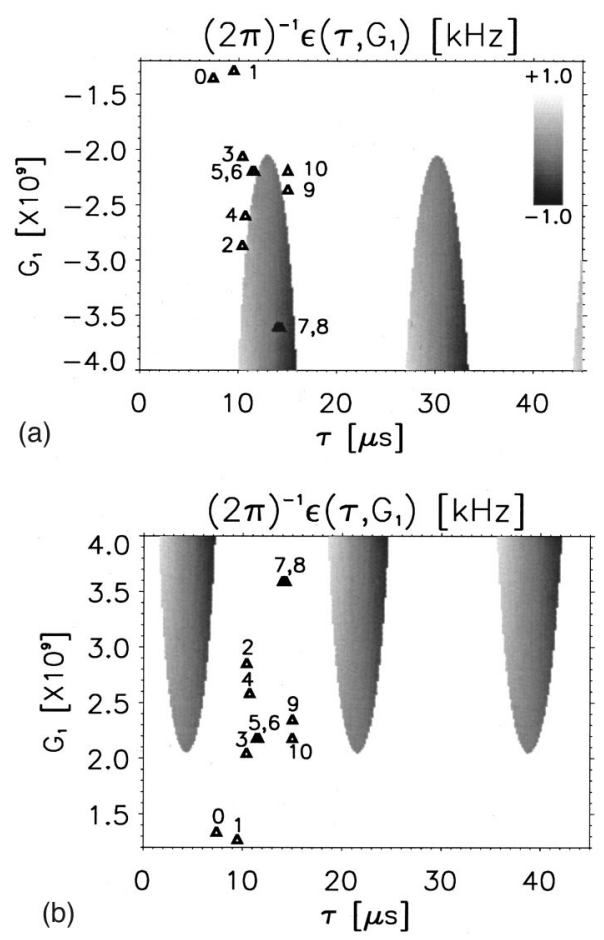

FIG. 4. Contours showing calculated values for $\epsilon\left(\tau, G_{1}\right)$ corresponding to the available values of $\alpha_{1}^{p}\left(\tau, G_{1}\right)$ for: (a) negative values of $G$ and (b) positive values of $G$. Contour values shown are corresponding to $f_{1}=58$ $\pm 1 \mathrm{kHz}$. Also shown as triangles and labeled by numbers $0-10$, are experimental data listed in Table I. The frequencies given by $f=f_{1}+\epsilon / 2 \pi$ corresponding to the local contour are to be compared with the experimental data.

\section{DISCUSSION}

We now proceed to compare the results obtained using the beam equation with that obtained from the point mass model and relate these to experimental observations. As pointed out in Sec. IV, an important characteristic of the solution obtained from the beam equation as displayed in Figs. 2 and 3, is the presence of bands of allowed values of $\tau$ and corresponding values of $G_{1}$ and $\epsilon$. The primary difference between Fig. $2(G<0)$ and Fig. $3(G>0)$ is that the values of $\tau$ are shifted by $T_{1} / 2=8.2(\mu \mathrm{s})$. As explained earlier this shift can exactly be accounted for by the difference in the response of the piezoelectric bimorph with respect to that of the cantilever motion. Comparison between the cal-

TABLE I. Experimental values for the time delay, the gain factor, and the corresponding resonant frequencies of a triangular microcantilever.

\begin{tabular}{crrrc}
\hline \hline Entries & $\tau(\mu \mathrm{s})$ & $|G|$ & $f(\mathrm{kHz})$ & $(2 \pi)^{-1} \epsilon(\mathrm{kHz})$ \\
\hline 0 & 7.4 & 0.40 & 59.6250 & 1.63 \\
1 & 9.5 & 0.38 & 59.6250 & 1.63 \\
2 & 10.4 & 0.85 & 60.3900 & 2.39 \\
3 & 10.4 & 0.61 & 61.1540 & 3.15 \\
4 & 10.7 & 0.77 & 60.3900 & 2.39 \\
5 & 11.4 & 0.65 & 61.1540 & 3.15 \\
6 & 11.6 & 0.65 & 61.9190 & 3.92 \\
7 & 14.0 & 1.07 & 62.6830 & 4.68 \\
8 & 14.3 & 1.07 & 62.6830 & 4.68 \\
9 & 15.0 & 0.70 & 62.6830 & 4.68 \\
10 & 15.0 & 0.65 & 62.6830 & 4.68 \\
\hline \hline
\end{tabular}



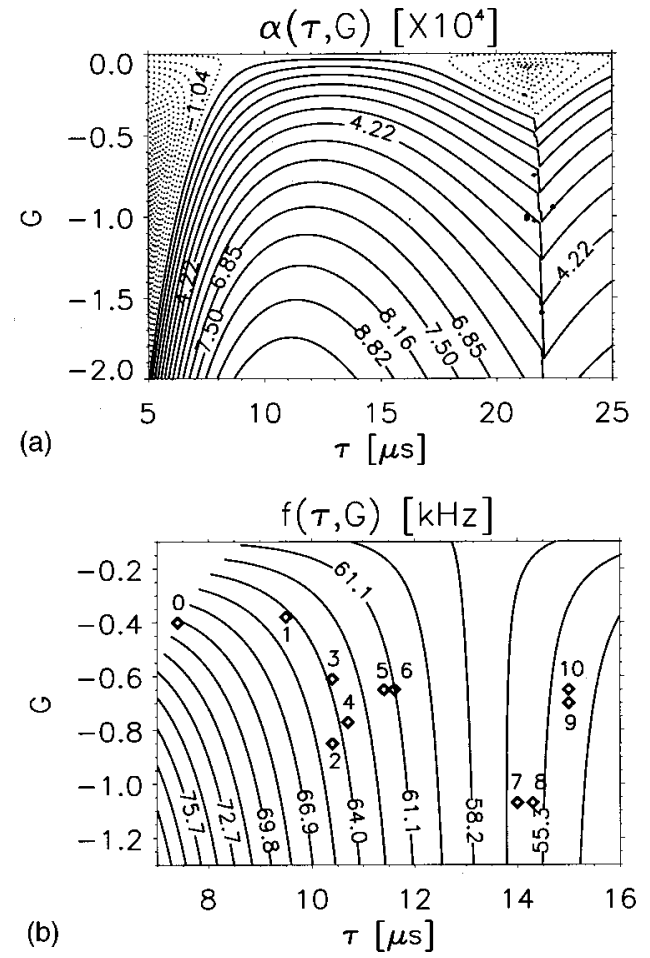

FIG. 5. (a) Variation in $\alpha_{1}^{p}(\tau, G)$ in the vicinity of the fundamental resonant frequency of the system $\omega_{1}$, as a function of delay and negative values of gain calculated using the point mass model. As before, the dotted lines represent the negative values of $\alpha(\tau, G)$ and are not considered. (b) Calculated values for $\epsilon(\tau, G)$ corresponding to the available values of the amplification $\alpha_{1}^{p}(\tau, G)$. Also shown are the experimentally observed combinations of $\tau$ and $|G|$ for which amplification was observed.

culated values of $\tau$ and experimental data shows that $G<0$ results in better agreement and thus will be discussed further.

Figures 4(a) and 4(b) show the frequencies corresponding to the allowed combinations of $\tau$ and $G$. If one considers the range of time delays from 5 to $25 \mu$ s, the values of $\tau$ for which amplification will succeed $(G<0)$ is restricted to the interval $10-16 \mu \mathrm{s}$ by the beam equation. For purpose of comparison, we display in Figs. 5(a) and 5(b), the calculated amplifications and resonant frequencies for the cantilever utilizing the point mass model. ${ }^{13,14}$ Although Fig. 5(a) displays scattered forbidden regions along with a discontinuity in the domain of available amplification close to $\tau \approx 22 \mu \mathrm{s}$, no further restriction is imposed on the time delay in this model. Thus, a wide range of $\tau$ values are feasible for various values of $G$, along with a large variation in the allowed frequencies.

Figures 4(a) and 5(b) also compare the predicted values of combinations of $\tau$ and $G$ obtained using the beam equation and the point mass model, respectively, with the experimentally observed values. Clearly except for the two points with low values of $\tau$, there is excellent agreement between the predictions using the beam equation and the experimental values of $\tau$ and $G$ for which amplification was observed. Most of the combinations are observed to be along the borders of the allowed region. This may be explained by the fact that experimentally, the regions were approached from either the low $\tau$ side or from the high $\tau$ side and hence resonance was attained for the first value of $\tau$ that allowed resonance. From Fig. 5(b) it should be noted that the experimentally
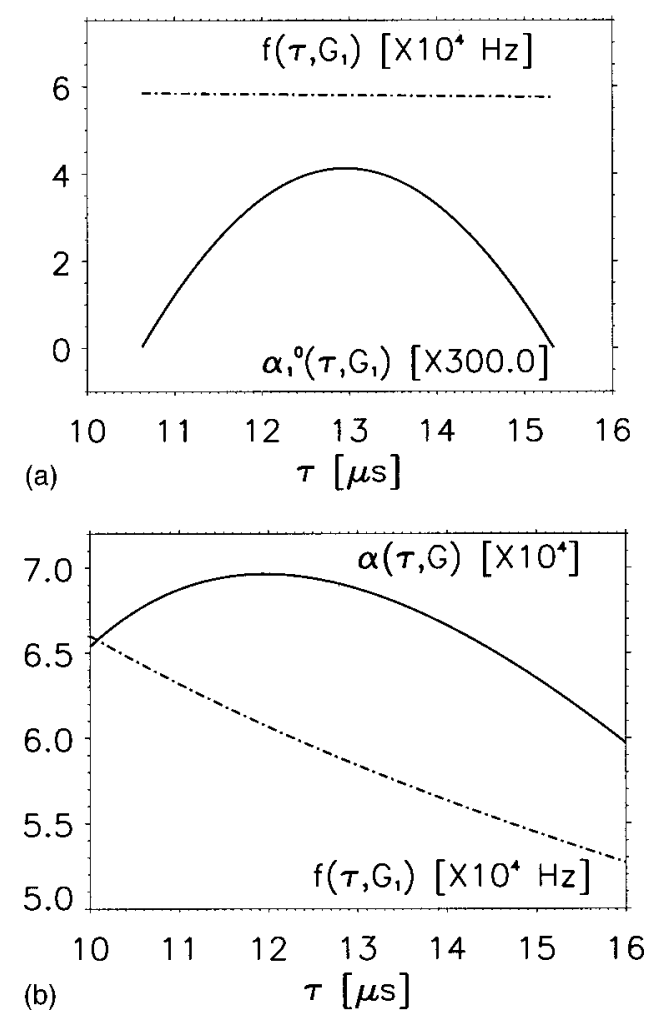

FIG. 6. (a) Variation in frequency $f\left(\tau, G_{1}\right)$ and amplification $\alpha\left(\tau, G_{1}\right)$ for a constant value of $G_{1}=-1.0$ as calculated using the beam model. As explained in the text $G_{1}$ has been normalized for comparison with the results obtained by using the point-mass model. (b) Functions $\alpha(\tau, G)$ and $f$ evaluated at $G=-1$ in Figs. 5(a) and 5(b).

observed combinations of $\tau$ and $G$ also fall on traces of allowed combinations of these two values. The agreement is less dramatic with this model, since the combinations of allowed values of $\tau$ and $G$ are much larger than in the case of the beam equation.

Important differences are observed between the two models with respect to the predicted frequencies. Calculations using the beam equation predict only a very small variation in the range of allowed frequencies over the entire range of $\tau$ values for which amplification succeeds. Figure 4(a) shows for example a variation in frequencies of 58.0 $\pm 1.0 \mathrm{kHz}$ over the entire range of allowed $\tau$ values. In contrast, the point mass model predicts a large range of allowed frequencies. Figure 5(b) shows that the predicted frequencies are approximately in the interval $[53.0,68.4]=58.0+(-5,10)$ $(\mathrm{kHz})$. Comparison with Table I shows that the experimentally observed frequencies range from 59.6 to $62.7 \mathrm{kHz}$ $(58.0+5 \mathrm{kHz})$ for all observed combinations of $\tau$ and $G$. Thus, the values of the frequencies predicted by using the beam equation are always smaller than the measured frequencies by about $3 \%-8 \%$. The frequencies predicted by the point mass can either be larger or smaller than the observed frequencies and differ by values of $-15 \%$ to $+15 \%$. Thus, the beam equation is significantly better in predicting the experimentally observed frequencies.

The differences between the two models are further illustrated in Figs. 6(a) and 6(b) which show a horizontal slice through Figs. 2 and 5, respectively, for a particular value of 
$G=G_{1} \approx-1$. Figure 6(a) shows a trace of the allowed values of amplification $\left(\alpha_{1} \geqslant 0\right)$ as a function of $\tau$ (in the first band $p=0$ ) and the corresponding frequencies obtained from the beam equation, while Fig. 6(b) shows the results obtained from the point mass model. To maintain consistency with the definitions of $G$ between the two models, the values of $G_{1}$ shown in Figs. 2(a) and 2(b) have to be scaled by a factor of $k^{\prime} / \mu=3.36 \times 10^{9}$ [see Eqs. (18) and (26)]. Figure 6(a) shows that the allowed frequencies are centered around the fundamental frequency $f_{1}=58 \mathrm{kHz}$ and vary linearly with $\tau$, with the variation being $\pm 0.5 \mathrm{kHz}$ or $57.4 \leqslant f \leqslant 58.5 \mathrm{kHz}$. However, the allowed frequencies for the point mass model vary nonlinearly with $\tau$ and range from a value of 52 to $66 \mathrm{kHz}$ for delays in the range $10 \leqslant \tau \leqslant 16 \mu \mathrm{s}$. The experimentally observed frequency for this combination of $\tau=14.0 \mu \mathrm{s}$ and $G=1.07$ was $f_{1}=62.68 \mathrm{kHz}$. Compare this with the predictions by using the beam equation $\left(f_{1}=57.4 \mathrm{kHz}\right)$ and the point mass model $\left(f_{1}=56.0 \mathrm{kHz}\right)$. Clearly, use of the beam equation results in a value better than that obtained by using the point mass model. However, it should be observed that both models predict a value smaller than the undriven frequency while the observed resonant frequency is larger than the undriven frequency.

Although the range of allowed values of time delays predicted by the two models is different, some features are similar between the two models. It can be seen from Figs. 6(a) and 6(b) that the amplification reaches a maximum in both models for comparable $\tau$ values. For the beam equation, the observed maximum in $\alpha_{1}^{0}$ at $\tau=12.97 \mu$ s (roughly $3 T_{1} / 4$ ) corresponds to $f=f_{1}=58.0 \mathrm{kHz}$ where $f_{1}$ is the fundamental frequency. Compare this with the predictions using the point mass model where the maximum value of $\alpha$ occurs at $\tau=11.96 \mu \mathrm{s}$ with a corresponding frequency of $f$ $=60.75 \mathrm{kHz}$. Reference to Table I shows that for $G=1.07$ the observed delays were $\tau=14$ and $\tau=14.3 \mu$ s with the corresponding frequency being $f=62.68 \mathrm{kHz}$. It is interesting to note that the frequency corresponding to the maximum value of $\alpha(f=60.75 \mathrm{kHz})$ in the point mass model corresponds better to the experimentally observed frequency of $(f=62.68 \mathrm{kHz})$ than that predicted for the appropriate value of $\tau=14.0 \mu \mathrm{s}(f=56.0 \mathrm{kHz})$.

Comparing the frequencies observed experimentally with those predicted by the models, it is clear that the use of the beam equation is more restrictive than is necessary, while the use of the point mass model does not sufficiently restrict the solutions. Two assumptions made in this work could be likely sources of the discrepancies in the observed and calculated range of allowed frequencies. It should be recalled that experimentally, the feedback signal results only from the instantaneous position of the tip of the cantilever. However, in Eq. (5), it is assumed that the feedback signal results from each point along the length of the cantilever and hence is much more restrictive than that necessary to model the experiments. Thus, Eq. (5) should be modified so as to address this issue in a more physically realistic manner while maintaining the mathematical difficulty associated with delay differential equations within a reasonable frame of work. Ongoing work is exploring numerical approaches to solve this problem. Additionally, to formulate the differential equations it has been assumed that cantilever mass is uniform along its length. However, the data reported in this and previous work have been obtained from triangular cantilevers for which the mass varies as a function of its length. Recent experiments suggest that the agreement is better for rectangular cantilevers and will be the subject of a forthcoming work.

In summary, we observe that the model utilizing the beam equation presented in this work is a significant improvement over the point mass model outlined in our earlier work. Criteria obtained using the beam equation agree well with the experimental values of $\tau$ and $|G|$ for which amplification in oscillations are observed in the feedback mode. It is also capable of predicting the observed frequencies with less absolute error than the point mass model. It is expected that improvements in the formulation of the beam model will result in a better agreement in the direction of change in frequency and further reduce the magnitude of error in the frequencies.

\section{CONCLUSIONS}

It has been shown that by taking into account the spatial extent of a microcantilever driven via a delayed feedback mechanism, successful amplification of its oscillations at frequencies close to its fundamental frequency can be predicted, given that the delay time and gain obey certain conditions. We have shown that meeting these conditions restricts the range of time delays to bounded intervals as observed experimentally, a behavior not predicted by the simple point mass model. In addition, the proposed model using the beam equation predicts a range of allowed frequencies that is much narrower than that predicted by the point mass model, in better agreement with the experimental results.

\section{ACKNOWLEGMENTS}

This work was supported by the DOE Office of Biological and Environmental Research (OBER). Oak Ridge National Laboratory, Oak Ridge, Tennessee is managed by UTBattelle, LLC for the Department of Energy under Contract No. DE-AC05-0096OR22725.

\section{APPENDIX}

To show that $K$ must be positive, disregarding the trivial solution $W(x, t) \equiv 0$, multiply the first equation in Eq. (8) by $X(x)$ and integrate over the length of the beam. Partial integration and the boundary conditions in Eq. (8) imply

$$
\begin{aligned}
\int_{0}^{1} X^{(4)}(x) X(x) d x & =\left.X^{(3)}(x) X(x)\right|_{0} ^{1} \\
& -\int_{0}^{1} X^{(3)}(x) X^{\prime}(x) d x \\
& =-\left.X^{\prime \prime}(x) X^{\prime}(x)\right|_{0} ^{1}+\int_{0}^{1}\left[X^{\prime \prime}(x)\right]^{2} d x \\
& =\int_{0}^{1}\left[X^{\prime \prime}(x)\right]^{2} d x .
\end{aligned}
$$


Thus

$$
\int_{0}^{1}\left\{\left[X^{\prime \prime}(x)\right]^{2}-\frac{K L^{4}}{E I}[X(x)]^{2}\right\} d x=0 .
$$

If $K \leqslant 0$, the above implies that $X^{\prime \prime}(x) \equiv 0$; that is, $X^{\prime}(x)$ $\equiv$ const. Now the condition $X^{\prime}(0)=0$ implies $X^{\prime}(x) \equiv 0$. So $X(x) \equiv$ const. Use the fact that $X(0)=0$ to conclude $X(x)$ $\equiv 0$, which is the trivial solution. Therefore, we may assume that $K>0$.

${ }^{1}$ G. Binnig, C. F. Quate, and Ch. Gerber, Phys. Rev. Lett. 56, 930 (1986).

${ }^{2}$ H. Takano, J. R. Kenseth, S. S. Wong, J. C. OBrien, and M. D. Porter, Chem. Rev. 99, 2845 (1999).

${ }^{3}$ B. Cappella and G. Dietler, Surf. Sci. Rep. 34, 1 (1999).

${ }^{4}$ D. Sarid, Scanning Force Microscopy with Applications to Electric, Magnetic, and Atomic Forces, Oxford Series on Optical Sciences (Oxford University Press, Oxford, 1991).

${ }^{5}$ G. Y. Chen, R. J. Warmack, T. Thundat, D. P. Allison, and A. Huang, Rev. Sci. Instrum. 65, 2532 (1994).
${ }^{6}$ J. E. Sader, J. Appl. Phys. 84, 64 (1998).

${ }^{7}$ U. Rabe, K. Janser, and W. Arnold, Rev. Sci. Instrum. 67, 3281 (1996).

${ }^{8}$ J. A. Turner, S. Hirsekorn, U. Rabe, and W. Arnold, J. Appl. Phys. 82, 966 (1997).

${ }^{9}$ J. W. M. Chon, P. Mulvaney, and J. E. Sader, J. Appl. Phys. 87, 3978 (2000).

${ }^{10}$ T. R. Albrech, P. Grtter, D. Horne, and D. Rugar, J. Appl. Phys. 69, 668 (1991).

${ }^{11}$ U. Dürig, H. R. Steinauer, and N. Blanc, J. Appl. Phys. 82, 3641 (1997).

${ }^{12}$ A. Mehta, S. Cherian, D. Hedden, and T. Thundat, Appl. Phys. Lett. 78, 1637 (2000).

${ }^{13}$ G. Muralidharan, A. Mehta, S. Cherian, and T. Thundat, J. Appl. Phys. 89, 4587 (2001).

${ }^{14}$ A. Passian, G. Muralidharan, S. Kouchekian, A. Mehta, S. Cherian, T. L. Ferrell, and T. Thundat (unpublished, 2001).

${ }^{15}$ K. F. Graff, Wave Motion in Elastic Solids (Dover Publications, New York, 1991).

${ }^{16}$ D. R. Bland, The Theory of Linear Viscoelasticity (Pergamon, New York, 1960).

${ }^{17}$ R. Bellman and K. L. Cooke, Differential-Difference Equations (Academic, New York, 1963). 